\title{
On a conformal mapping with certain boundary correspondences.
}

Akira MORI.

(Received, Oct. 1, 1949)

Given any set of points $E$ on the unit circle $C$ of $z$-plane, we shalf treat in this paper the problem to map the interior of $C$ conformally on a schlicht domain $D$ so that the set $E$ corresponds to a set of accessibleboundary points of $D$ all lying on one and the same point of the plane.

In order to simplify the wording, we call a half straight-line on $w$ plane: $\arg w=$ const., $\infty \geqq|w| \geqq$ const. $>0$, an infinite radial slit.

First, we consider the case where the set $E$ is finite.

Theorem 1. Let $z_{1}, \ldots, z_{n}$ be $n$ points on $C$ associated rvith $n$ positive numbers $\alpha_{1}, \ldots, \alpha_{n}$, whose sum is equal to 1 . Then, there exists a function $w=$ $z u(z)$, which maps the interior of $C$ conformally on a domain $D$, so that $: 1$. $D$ is the whole ze-plane cut along $n$ infinite radial slits, $2 . z_{k}$ corresponds to the accessible boundary point of D lying on $w=\infty$, which is determined by an angular domain between two of these slits including an angle $2 \pi \mu_{k}$ at w $=\infty$, and $3 . \quad w(0)=0, w^{\prime}(0)=1$. Under these conditions the mapping function is uniquely determined and is given by

$$
\tau v=\tau v(z)=z \prod_{k=1}^{n}\left(1-\frac{z}{z_{k}}\right)^{2 a_{k}}
$$

Proof. We construct a potential function $u(z)$ on $z$-plane, whose singularities are

and

$$
\begin{aligned}
& \log |z| \quad \text { at } \quad z=0, \\
& \log \frac{1}{|z|} \text { at } z=\infty
\end{aligned}
$$

$$
2 \alpha_{k} \log \frac{1}{\left|z-z_{k}\right|} \text { at } z=z_{k}
$$

Denoting the conjugate potential of $u(z)$ by $v(z)$, we put

$$
w(z)=\text { const. exp. }\{u(z)+i v(z)\} \text {. }
$$


$w(z)$ is regular in $|z| \leqq 1$ except the $n$ points $z_{k}$, and vanishes only at $z=0$.

On account of symmetry of singularities, $u(\varepsilon)$ takes one and the same value at $z$ and its reflection $1 / \bar{z}$ in $C$. Hence, we have, putting $z=r e^{i \theta}$, at any point on $C$ except $z_{1}, \ldots \ldots, z_{n}$,

$$
\frac{\partial u}{\partial r}=0 \quad \text { consequently } \frac{\partial v}{\partial \theta}=0 .
$$

which means that $v(z)$ is equal to a constant on each of $n$ arcs of $C$ separated by the $n$ points $z_{k}$. On the other hand, $u(z)$ is bounded below and unbounded above on each of these arcs. Hence the image of each of these arcs by $w=w(z)$ is an infinite radial slit.

The angle at $v=\infty$ between the images of two arcs forming an angle $\pi$ at $z_{k}$ is equal to $2 \pi u_{k}$, since $z(z)$ has an expansion of the form

$$
\left(z-z_{k}\right)^{-2 a_{k}}\left\{C_{0}+C_{1}\left(z-z_{k}\right)+\ldots \ldots\right\} \quad\left(C_{0} \neq 0\right)
$$

in a neighbourhood of $z=z_{k}$.

Further, $z(z)$ takes each value $z v_{0}$, which does not belong to the $n$ slits, once and only once in $|z|<1$. This follows easily from the facts that $1 / \approx v(z)$ has only one pole in $|z|<1$ and that

$$
\arg \left\{\frac{1}{z v(z)}-\frac{1}{z v_{0}}\right\}
$$

remains unchanged, when $z$ moves on $C$ once around and returns to the original value. Hence, by suitable determination of constant factor, $w(z)$ constructed above provides the required mapping.

Since the potential function $u(z)$ with required singularities is explicitly given by

$$
u(z)=\sum_{k=1}^{n} 2 \alpha_{k} \log \frac{1}{\left|z-z_{k}\right|}+\log |z|+\text { const., }
$$

we have the mentioned expression (1) for $w(z)$.

The uniqueness of the mapping function is proved as follows. Let $z v_{1}(z)$ be another mapping function with the properties 1,2,3. Since $\left|w_{1}\right|$ remains unchanged by reflection in a radial slit, we obtain a one-valued harmonic 
function $\log \left|w_{1}(z)\right|$ with isolated singularities at $z=0, \infty$ and $z_{1}, \ldots \ldots, z_{n}$, while continuing $w_{1}(z)$ analytically across the unit circle by the principle of reflection. Then, by the expansion (2), $\log \left|w_{1}(z)\right|$ must be identical with $\log |w(z)|$, save for an additive constant.

We know, if $z(z)$ is schlicht and star-shaped in $|z|<1$ with respect to $w(0)=0$ being normalised by $w(0)=1$, it is expressed in the form

$$
\tau(z)=z \cdot \exp \cdot 2 \int_{c} \log \frac{\zeta}{\zeta-z} d \mu(\zeta)
$$

and vice versa, where $\mu$ is a positive distribution of total mass 1 on $C$ determined by the non-decreasing function of $\theta$

$$
\frac{1}{2 \pi} \lim _{r \rightarrow 1} \arg i v\left(r e^{t \theta}\right) \text {. }
$$

This can easily be proved by applying Herglotz' formula to $z w / \tau u$ and integrating it.

The mapping function (1) of Theorem 1 is in fact merely a special case of this formula, where $\mu$ vanishes ontside the $n$ points $z_{1}, \ldots \ldots, z_{n}$.

Next, we consider the case where the given set $E$ is infinite: In case $E$ consists of an enumerable infinity of points, we can construct a mapping function analogous to that of Theorem 1 by taking the limes of functions of the form (1), or, simply by (3), while we give a positive $\mu$-mass to each point of $E$. But then, the boundary of the resulting image domain is in general very much complicated.

No matter whether $E$ be enumerable or not, we have in the following case an image domain whose boundary is fairly simple.

Theorem 2. If the closure $\bar{E}$ of $E$ is of logarithmic capacity zero, and only in such a case, there exists a function w(z), which maps the interior of $C$ conformally on a domain $D$, so that: $1 . D$ is the whole w-plane cut along an enumerable infinity of infinite radial slits, auhich cluster to $z=\infty$ only, 2. every point of $E$ corresponds to an accessible boundary point of $D$ lying on $w=\infty$, and 3. $w(0)=0$.

Proof. If $\bar{E}$ is of logarithmic capacity zero, there exists, by Evans' theorem, ${ }^{1)}$ a positive distribution $\mu$ of total mass 1 on $\bar{E}$, such that the logarithmic potential

$$
\int_{\bar{E}} \log \frac{1}{|\zeta-z|} d \mu(\zeta)
$$


tends to. $+\infty$, when $z$ tends to any point of $\bar{E}$. Then the star-shaped function (3) constructed with this $\mu$ tends to $\infty$, whenever $z$ tends to $\bar{E}$, and provides the required mapping.

On the contrary, if $\bar{E}$ is of positive capacity, there exists, for any positive mass distribution $\mu$ on $C$, at least one point $\zeta_{0}$ on $\bar{E}$, such that

$$
\lim _{z \rightarrow \zeta_{0}} \int_{c} \log \frac{1}{|\zeta-z|} d \mu(\zeta)<+\infty
$$

and we have

$$
\lim _{z \rightarrow \zeta_{0}}|\tau(z)|<+\infty .
$$

On the other hand, if $z(z)$ satisfies the condition 2 , we have

$$
\varlimsup_{z \rightarrow \zeta_{0}}|z e(z)|=+\infty
$$

for any point $\zeta_{0}$ on $\bar{E}$, so that it can not satisfy the condition 1 .

Let $\Delta$ be a simply connected schlicht domain, and $e$ be a closed set of accessible boundary points of $\Delta$, which is of logarithmic capacity zero. Then M. Tsuji ${ }^{2)}$ proved the following extention of Beurling's theorem ${ }^{3)}$ on exceptional sets : if we map $\Delta$ conformally on the interior of the unit circle $C$, then the set $E$ of points on $C$, which corresponds to the set $e$, is of logarithmic capacity zero.

By this theorem, we have from Theorem 2 the following

Theorem 3. If each primary end of $\Delta$ in Caratheodory's sense, zuhich contains a point of $e$, consists of only one point, then we can map $\Delta$ conformally on a domain $D$ satisfying the condition 1 of Theorcm 2, so that each point of e corresponds to the point at infinity. .

Proof. From the hypothesis we see easily that $E$ is closed, so that the result follows from Tsuji's theorem and Theorem 2.

\section{Mathematical Institute, \\ Tokyo University.}

\section{References.}

1) G. C. Evans: Potentials and positively infinite singularities of harmonic functions. Monatshefte f. Math. u. Phys. 43 (1936).

2) M. Tsuji : Beurling's theorem on exceptional sets (which will appear in Tohoku Math. J.).

3) A. Beurling: Ensembles exceptionnels. Acta Math. 72 (1940). 